\title{
Incidence, Microbiological Profile and Risk Factors of Healthcare-Associated Infections in Intensive Care Units: A 10 Year Observation in a Provincial Hospital in Southern Poland
}

\author{
Małgorzata Kołpa ${ }^{1}$, Marta Wałaszek ${ }^{1}$, Agnieszka Gniadek ${ }^{2, *}$, Zdzisław Wolak $^{1}$ and \\ Wiesław Dobroś ${ }^{1}$ \\ 1 The Institute of Health Sciences, State Higher Vocational School in Tarnów, ul. Mickiewicza 8, \\ 33-100 Tarnów, Poland; malgorzatakolpa@interia.pl (M.K.); mz.walaszek@gmail.com (M.W.); \\ zdzich_w@interia.pl (Z.W.); wdobros@wp.pl (W.D.) \\ 2 Department of Nursing Management and Epidemiology Nursing, Faculty of Health Sciences, \\ Jagiellonian University Medical College, ul. Kopernika 25, 31-501 Kraków, Poland \\ * Correspondence: agnieszka.gniadek@uj.edu.pl; Tel.: +48-124-214-010; Fax: +48-124-294-872
}

Received: 5 December 2017; Accepted: 8 January 2018; Published: 11 January 2018

\begin{abstract}
Healthcare-associated infections (HAIs) occurring in patients treated in an intensive care unit (ICU) are serious complications in the treatment process. Aetiological factors of these infections can have an impact on treatment effects, treatment duration and mortality. The aim of the study was to determine the prevalence and microbiological profile of HAIs in patients hospitalized in an ICU over a span of 10 years. The active surveillance method was used to detect HAIs in adult patients who spent over $48 \mathrm{~h}$ in a general ICU ward located in southern Poland between 2007 and 2016. The study was conducted in compliance with the methodology recommended by the Healthcare-associated Infections Surveillance Network (HAI-Net) of the European Centre for Disease Prevention and Control (ECDC). During the 10 years of the study, 1849 patients hospitalized in an ICU for a total of 17,599 days acquired 510 with overall HAIs rates of $27.6 \%$ and $29.0 \%$ infections per 1000 ICU days. Intubation-associated pneumonia (IAP) posed the greatest risk (15.2 per 1000 ventilator days), followed by CLA-BSI (8.0 per 1000 catheter days) and CA-UTI (3.0 per 1000 catheter days). The most common isolated microorganism was Acinetobacter baumannii (25\%) followed by Coagulaase-negativ staphylococci (15\%), Escherichia coli (9\%), Pseudomonas aeruginosa $(8 \%)$, Klebsiella pneumoniae (7\%), Candida albicans (6\%). Acinetobacter baumannii in $87 \%$ and were classified as extensive-drug resistant (XDR). In summary, in ICU patients pneumonia and bloodstream infections were the most frequently found. Acinetobacter baumannii strains were most often isolated from clinical materials taken from HAI patients and showed resistance to many groups of antibiotics. A trend of increasing resistance of Acinetobacter baumannii to carbapenems was observed.
\end{abstract}

Keywords: healthcare-associated infections; hospital infection intensive care unit; bloodstream infection urinary tract infection; ventilator-associated pneumonia; antibiotic resistance; Acinetobacter baumannii

\section{Introduction}

According to multiple sources, between $20 \%$ and $50 \%$ of healthcare-associated infections (HAIs) occur in the intensive care unit (ICU). In 2007, 39\% of patients in European countries were affected by these infections [1]. In Poland HAIs were detected in $24-45 \%$ of ICU patients [2,3]. Meta-analysis of HAIs showed that a HAIs affected $30 \%$ of patients [4]. In Italy frequency of HAIs was lower $(16 \%)$ [5]. These infections presented in the most dangerous and sometimes life-threatening forms of 
infection, such as pneumonia, bloodstream infection, and urinary tract infection; therefore, surveillance of these types of infections is important in the ICU [6,7]. The prevention of these infections is correlated with high treatment costs and longer hospitalization times [8]. Among healthcare-associated infections, PN is the most common and accounts for between $30 \%$ and $50 \%$ of all infections [6,8-11]. Epidemiological studies indicate that bloodstream infections are low frequently found and their prevalence ranges from $4 \%$ to $20 \%[6,8,10,11]$. Among HAIs, the prevalence of urinary tract infections ranges from $3 \%$ to $7 \%[8,10,11]$. Studies by Gordats et al. [10] showed that, among individuals with reduced immunity, which included ICU patients, other forms of HAIs also appeared.

The development of HAIs can be facilitated by the ICU environment, medical procedures applied to treat the patient and, above all, the patient's general health. Intensive care units treat severely ill patients whose underlying disease and coexisting diseases may contribute to the development of healthcare-associated infections $[10,12]$. The need to perform many diagnostic and treatment activities on patients results in the use and long-term maintenance of invasive devices, such as central vascular lines (CVC), intubation tubes, and urethral catheters (which may result in the removal of the natural protective barrier against infections [13]. Patients' stays in the ICU are often preceded by hospitalization in other hospital wards, during which there are often problems with the treatment of such patients. During the patient's stay in hospital (regardless of the ward in which they are hospitalized), the bacterial physiological microbiota are replaced with the hospital microbiota typical of a given ward. In addition, long-term antibiotic treatment leads to the selection of sensitive microorganisms in the patient's organisms, which may facilitate a conquest of the organism by pathogenic microorganisms (also by those coming from the environment) with a proven mechanism of resistance to antibiotics $[13,14]$. Therefore, it is extremely important to undertake actions that will at least protect patients from the negative health effects of microorganisms coming from a hospital environment; this is especially true for patients in intensive care units, where the invasiveness of treatments and reduced immunity of the patient will predispose them to an increased number of colonizations and infections. The results of the analysis demonstrated below are among few such studies in Poland and can be used as a reference to the actual epidemiological situation prevailing in ICU units of other hospitals in Poland, Europe or other continents. The aim of the study was to determine the incidence and microbiological profile of healthcare-associated infections in an intensive care unit.

\section{Materials and Methods}

The active surveillance method was used to detect HAIs in adult patients who spent over $48 \mathrm{~h}$ in a 9-bed general ICU ward located in southern Poland between 2007 and 2016. The ward under study was part of a multi-profile hospital (670 hospital beds). Not all patients included in the study were examined with the application of SOFA and APACHEII scales, therefore these scales were not taken under consideration. Definitions developed by the ECDC were used to identify and qualify infections [6,7]. The data collection methodology was fully compliant with the European Centre for Disease Prevention and Control (ECDC) recommended by the Healthcare-associated Infections Surveillance Network (HAI-Net) [7]. The HAI study was divided into the main and specific types of HAIs, such as BSI, PN, UTI, and OTH (other infections). Among the OTH infections, the following were distinguished: CNS, EENT, LRI, SYS.

Bloodstream infection was defined as a patient has at least one positive blood culture for a recognised pathogen or patient has at least one of the following signs or symptoms: fever $\left(>38^{\circ} \mathrm{C}\right)$, chills, or hypotension and two positive blood cultures for a common skin contaminant (from two separate blood samples, usually within 48 h). Skin contaminants = coagulase-negative staphylococci, Micrococcus spp., Propionibacterium acnes, Bacillus spp., Corynebacterium spp. [7].

Pneumonia (PN1-PN5) was defined as two or more serial chest X-rays or CT-scans with a suggestive image of pneumonia for patients with underlying cardiac or pulmonary disease. In patients without underlying cardiac or pulmonary disease, one definitive chest X-ray or CT-scan is sufficient. And at least one of the following: fever $>38^{\circ} \mathrm{C}$ with no other cause leukopenia $\left(<4000 \mathrm{WBC} / \mathrm{mm}^{3}\right)$ 
or leucocytosis $\left(\geq 12,000 \mathrm{WBC} / \mathrm{mm}^{3}\right)$ and at least one of the following (or at least two, if clinical pneumonia only = PN4 and PN5): new onset of purulent sputum, or change in character of sputum (colour, odour, quantity, consistency), cough or dyspnea or tachypnea suggestive auscultation (rales or bronchial breath sounds), rhonchi, wheezing, worsening gas exchange (e.g., $\mathrm{O}_{2}$ desaturation or increased oxygen requirements or increased ventilation demand) and according to the used diagnostic method [7].

Urinary tract infection (UTI) was defined as a microbiologically confirmed symptomatic urinary tract infection where the patient has at least one of the following symptoms with no other recognised cause: fever $\left(>38^{\circ} \mathrm{C}\right)$, urgency, frequency, dysuria, or suprapubic tenderness and patient has a positive urine culture, i.e., $\geq 105$ microorganisms per $\mathrm{mL}$ of urine with no more than two species of microorganisms [7].

A device-associated, healthcare-associated infection is an HAI in a patient with a (relevant) device that was used within a 48 -h period before the onset of infection. The term 'deviceassociated' is only used for pneumonia, bloodstream infections, and urinary tract infections. 'Relevant devices' refers to intubation, central vascular catheters, and urinary catheters. If the interval is longer than $48 \mathrm{~h}$, there must be compelling evidence that the infection was associated with the device use. For catheter-associated UTI, an indwelling urinary catheter must have been in place seven days before positive laboratory results or signs and symptoms meeting the criteria for UTI were evident. Example: Pneumonia is defined as intubation-associated (IAP) if an invasive respiratory device was present (even intermittently) in the $48 \mathrm{~h}$ preceding the onset of infection [7].

Blood infections were monitored as Catheter Related Infections (CRIs) including the division into types of catheters used and microbiological diagnosis.

The results of microbiological studies performed with clinically relevant materials, such as blood, urine, smear from wounds, faeces, broncho-aspirate, vascular lavage, vascular catheter tips and others, were interpreted. The hospital applied the principles of the European Committee on Antimicrobial Susceptibility Testing (EUCAST). Strains resistant to antibiotics were defined in the following way: Extensive Drug Resistant (XDR)—resistant to one or more antibiotics in all groups of antibiotics; multi drug resistant (MDR)—resistant to at least one antibiotic from three or more groups of antibacterial drugs active for a particular type of microbial genus or Pan Drug Resistant (PDR) - resistant to all antibiotics in all groups of antibiotics active for a particular genus. Microbial cultures were incubated on chromogenic media: Chromid ID CPS, Columbia with $5 \%$ of ram blood, Sabouraud agar, Schaedler agar, trypicase soy agar, chocolate agar, MacConkey agar, Hektoen enteric agar, sodium selenate agar. Microorganisms were identified by means of biochemical tests applied in automatic system of identification: Vitek 2 Compact (bioMérieux, Marcy-l'Étoile, France).

The following epidemiological indicators were used to investigate the epidemiological situation: cumulative incidence and incidence density. The incidence $=$ number of $\mathrm{HAI} \times 100$ per number of patients. The incidence density of HAIs per 1000 days of device use. Density CLA-BSI = number of CLA-BSI $\times 1000$ per number of central line-days. Density IAP $=$ number of IAP $\times 1000$ per number of intubation-days (VAP w CDC/INICC). Density CA-UTI = number of CA-UTI $\times 1000$ per number of urinary catheter-days. Mortality (\% death of HAIs = number of deaths HAI $\times 100$ per number of HAI). Device utilization ratio (DUR) = number of an invasive device days (CVC, intubation, urinary catheter) per number of patient days. All the data that were entered into an electronic database and analyses in this study were anonymized previously.

Statistical Package for the Social Sciences (SPSS STATISTICS 24, Armonk, NY, USA) and Microsoft Excel Microsoft Office 2016 (Redmond, WA, USA) were used in the statistical analysis of the collected material. The significance level was $p<0.05$. In addition, the pooled mean, median (Me), standard deviation (SD), 95\% confidence interval for mean (95\% CI), odds ratio (OR), Chi-squared (Pearson) $\chi^{2}$ for the ordinal and nominal scales and an ANOVA for the quantitative scale were calculated. The invasive procedures performed on patients were analyses as risk factors for HAI 
formation and calculated for Chi-squared (Pearson)'s materiality level, the quotient of chances and Pearson's correlations.

\section{Results}

During the 2007-2016 period 1847 patients were treated in the ICU, for more than $48 \mathrm{~h}$ each; 710 patients (38.4\%) were females, and 1137 patients $(61.6 \%)$ were males. The HAI risk was statistically significantly higher for men $(p<0.001)$ than for women, and the odds of HAI (OR) were greater for men than for women and amounted to 1.55 (95\% CI 1.24-1.92). The average age of patients hospitalized in the ICU was 58 (median 61 years, SD 20, and 95\% CI 57-59). Patients with HAIs were younger and were, on average, 54 years old (median 57 years, SD 18.495, and 95\% CI 52.8-56.1) than patients without HAIs, who were on average 59 years old (median age 62, SD 19.840, 95\% CI 58.2-60.2); this difference was statistically significant $(p<0.001)$.

In the group of 1847 patients under survey, 510 cases of HAI were diagnosed, and the HAI incidence rate was $27.6 \%$ in 100 patients admitted to the ICU. The main types of HAI detected in ICU patients included the following: PN (9.9\% incidence), BSI ( $8.9 \%$ incidence) and UTI ( $2.9 \%$ incidence). The central line-associated bloodstream infection (CA-BSI) incidence density was 8.0/1000 catheter-days, the device use ratio (DUR) was 0.96 , the ventilator-associated pneumonia (VAP) incidence density was 15.2/1000 ventilator-days, (DUR 0.65) and the catheter-associated urinary tract infection (CA-UTI) incidence density was 3.0/1000 catheter-days. In the material under examination, other types of HAI accounted for $22 \%$ of all infections, and the percentage of these infections was similar in all the years under analysis (Table 1 ).

Table 1. Number of HAIs/100 admissions (\%), number of HAIs/1000 person days $(n / 1000)$, person days $(n)$ by major and specific sites of infection in an intensive care unit in the years 2007-2016.

\begin{tabular}{|c|c|c|c|c|c|c|}
\hline HAI Types & $N$ & $\begin{array}{c}\text { Incidence } \\
\text { (\%) per } 100 \\
\text { Admissions } \\
\text { to ICU }\end{array}$ & $\begin{array}{c}\text { Incidence } \\
\text { Density per } \\
1000 \text { Days in } \\
\text { ICU }\end{array}$ & $\begin{array}{c}\text { Number of } \\
\text { Person Days } \\
\text { with } \\
\text { Invasive } \\
\text { Device* }\end{array}$ & $\begin{array}{l}\text { Incidence } \\
\text { Density per } \\
1000 \text { Person } \\
\text { Days with } \\
\text { Device }\end{array}$ & DUR \\
\hline All HAIs & 510 & 27.6 & 29.0 & & & \\
\hline Pneumonia (PN) & 183 & 9.9 & 10.4 & & & \\
\hline $\begin{array}{l}\text { PN1: minimally } \\
\text { contaminated LRT } \\
\text { sample with quantitative } \\
\text { culture }\end{array}$ & 13 & 0.7 & 0.7 & \multirow{5}{*}{12,059} & \multirow{5}{*}{$15.2 *$} & \multirow{5}{*}{0.65} \\
\hline $\begin{array}{l}\text { PN2: non-protected } \\
\text { sample with quantitative } \\
\text { culture }\end{array}$ & 14 & 0.8 & 0.8 & & & \\
\hline $\begin{array}{l}\text { PN3: alternative } \\
\text { microbiological criteria }\end{array}$ & 6 & 0.3 & 0.3 & & & \\
\hline $\begin{array}{l}\text { PN4: sputum } \\
\text { bacteriology or } \\
\text { non-quantitative }\end{array}$ & 145 & 7.9 & 8.2 & & & \\
\hline $\begin{array}{l}\text { PN5: no microbiological } \\
\text { documentation }\end{array}$ & 5 & 0.3 & 0.3 & & & \\
\hline $\begin{array}{l}\text { Bloodstream } \\
\text { infections (BSI) }\end{array}$ & 159 & 8.6 & 9.0 & & & \\
\hline $\begin{array}{l}\text { BSI-C: Primary BSI } \\
\text { catheter-related }\end{array}$ & 24 & 1.3 & 1.4 & \multirow{2}{*}{17,858} & \multirow[b]{2}{*}{$8.0^{* *}$} & \multirow[b]{2}{*}{0.96} \\
\hline $\begin{array}{l}\text { BSI-UO: Primary BSI of } \\
\text { unknown origin }\end{array}$ & 118 & 6.4 & 6.7 & & & \\
\hline
\end{tabular}


Table 1. Cont.

\begin{tabular}{|c|c|c|c|c|c|c|}
\hline HAI Types & $N$ & $\begin{array}{c}\text { Incidence } \\
(\%) \text { per } 100 \\
\text { Admissions } \\
\text { to ICU }\end{array}$ & $\begin{array}{c}\text { Incidence } \\
\text { Density per } \\
1000 \text { Days in } \\
\text { ICU }\end{array}$ & $\begin{array}{c}\text { Number of } \\
\text { Person Days } \\
\text { with } \\
\text { Invasive } \\
\text { Device * }\end{array}$ & $\begin{array}{l}\text { Incidence } \\
\text { Density per } \\
1000 \text { Person } \\
\text { Days with } \\
\text { Device }\end{array}$ & DUR \\
\hline BSI-S: secondary BSI & 17 & 0.9 & 1.0 & $\mathrm{n} / \mathrm{a}$ & $\mathrm{n} / \mathrm{a}$ & $\mathrm{n} / \mathrm{a}$ \\
\hline $\begin{array}{l}\text { Urinary tract } \\
\text { infection (UTI) }\end{array}$ & 54 & 2.9 & 3.1 & & & \\
\hline $\begin{array}{l}\text { UTI-A: microbiologically } \\
\text { confirmed symptomatic }\end{array}$ & 54 & 2.9 & 3.1 & 18,192 & $3.0 * * *$ & 0.98 \\
\hline $\begin{array}{l}\text { UTI-B: not } \\
\text { microbiologically } \\
\text { confirmed symptomatic }\end{array}$ & 0 & 0,0 & 0.0 & & & \\
\hline Gastrointestinal (GI) & 38 & 2.1 & 2.2 & & & \\
\hline $\begin{array}{l}\text { GI-CDI: clostridium } \\
\text { difficile infection }\end{array}$ & 20 & 1.1 & 1.1 & $\mathrm{n} / \mathrm{a}$ & $\mathrm{n} / \mathrm{a}$ & $\mathrm{n} / \mathrm{a}$ \\
\hline GI-GE: gastroenteritis & 18 & 1.0 & 1.0 & $\mathrm{n} / \mathrm{a}$ & $\mathrm{n} / \mathrm{a}$ & $\mathrm{n} / \mathrm{a}$ \\
\hline $\begin{array}{l}\text { Surgical site } \\
\text { infection (SSI) }\end{array}$ & 33 & 1.8 & 1.9 & & & \\
\hline SSI-D: Deep incisional & 18 & 1.0 & 1.0 & $\mathrm{n} / \mathrm{a}$ & $\mathrm{n} / \mathrm{a}$ & $\mathrm{n} / \mathrm{a}$ \\
\hline SSI-O: Organ/space & 6 & 0.3 & 0.3 & $\mathrm{n} / \mathrm{a}$ & $\mathrm{n} / \mathrm{a}$ & $\mathrm{n} / \mathrm{a}$ \\
\hline $\begin{array}{l}\text { SSI-S: Superficial } \\
\text { incisional }\end{array}$ & 9 & 0.5 & 0.5 & $\mathrm{n} / \mathrm{a}$ & $\mathrm{n} / \mathrm{a}$ & $\mathrm{n} / \mathrm{a}$ \\
\hline $\begin{array}{l}\text { Skin and soft tissue } \\
\text { infection (SST) }\end{array}$ & 25 & 1.4 & 1.4 & & & \\
\hline SST-DECU: soft tissue & 17 & 0.9 & 1.0 & $\mathrm{n} / \mathrm{a}$ & $\mathrm{n} / \mathrm{a}$ & $\mathrm{n} / \mathrm{a}$ \\
\hline SST-SKIN: skin infection & 5 & 0.3 & 0.3 & $\mathrm{n} / \mathrm{a}$ & $\mathrm{n} / \mathrm{a}$ & $\mathrm{n} / \mathrm{a}$ \\
\hline SST-ST: soft tissue & 3 & 0.2 & 0.2 & $\mathrm{n} / \mathrm{a}$ & $\mathrm{n} / \mathrm{a}$ & $\mathrm{n} / \mathrm{a}$ \\
\hline Other infections (OTH) & 18 & 1.0 & 1.0 & & & \\
\hline
\end{tabular}

The analysis identified risk factors, which included invasive procedures and potentially a significant factor in HAI formation. Statistically significant differences occurred between patients without and with HAIs in the following invasive procedures: mechanical ventilation, urethral catheter, administration of blood and plasma, pleural drainage, tracheostomy, percutaneous endoscopic gastrostomy (PEG), bronchoscopy, parenteral nutrition, and gastroscopy. Poor impact strength (Pearson's correlation) was found for the administered blood, plasma, pleural drainage, PEG probes, bronchoscopy and gastroscopy, indicating that the tendency to acquire HAI is evident with the use of these devices; there are, however, deviations. Moderate dependence was detected in the case of a patient who had tracheostomy, in which case the tendency to acquire an HAI was most visible (Table 2). 
Table 2. HAI risk factors in the ICU, 2007-2016.

\begin{tabular}{|c|c|c|c|c|c|c|}
\hline \multirow[t]{2}{*}{ Risk Factor } & Patients without HAI & $\begin{array}{c}\text { HAI } \\
\text { Patients }\end{array}$ & Total & \multirow[t]{2}{*}{ OR $(95 \%$ CI) } & \multirow[t]{2}{*}{$p$} & \multirow[t]{2}{*}{$\begin{array}{l}\text { Pearson's } \\
\text { Correlation }\end{array}$} \\
\hline & $n(\%)$ & $n(\%)$ & $n(\%)$ & & & \\
\hline Arterial catheter & $443(71.0 \%)$ & $181(29.0 \%)$ & $624(100 \%)$ & $1.1(0.9-1.4)$ & 0.595 & 0.0 \\
\hline Intubation & $287(86.2 \%)$ & $134(31.8 \%)$ & $421(100 \%)$ & $1.3(1.0-1.7)$ & 0.265 & 0.1 \\
\hline Mechanical ventilation & $887(69.8 \%)$ & $383(30.2 \%)$ & $1270(100 \%)$ & $1.5(1.2-1.9)$ & $<0.05$ & 0.1 \\
\hline Stomach probe & $447(71.0 \%)$ & $183(29.0 \%)$ & $630(100 \%)$ & $1.1(0.9-1.4)$ & 0.884 & 0.0 \\
\hline Blood & $310(53.0 \%)$ & $275(47.0 \%)$ & $585(100 \%)$ & $3.9(3.1-4.8)$ & $<0.001$ & 0.3 \\
\hline Pleural drainage & $67(46.5 \%)$ & $77(53.5 \%)$ & $144(100 \%)$ & $3.4(2.4-4.8)$ & $<0.001$ & 0.2 \\
\hline Tracheostomy & $98(27.9 \%)$ & $253(72.1 \%)$ & $351(100 \%)$ & $12.4(9.5-16.3)$ & $<0.001$ & 0.5 \\
\hline Peg & $38(30.6 \%)$ & $86(69.4 \%)$ & $124(100 \%)$ & $6.9(4.7-10.3)$ & $<0.001$ & 0.3 \\
\hline Plasma & $196(54.0 \%)$ & $167(46.0 \%)$ & $363(100 \%)$ & $2.8(2.2-3.6)$ & $<0.001$ & 0.2 \\
\hline
\end{tabular}

Chi-square (p); Odds ratio (OR); 95\% confidence interval (CI); percutaneous endoscopic gastrostomy (PEG).

A total of $93 \%$ of HAIs were caused by micro-organisms that were determined by being identified as a genus or species. A total of $7 \%$ of HAI infections were not microbiologically confirmed. BSI was dominated by Coagulase-negative staphylococci (44\%), PN was dominated by non-fermenting gram-negative bacteria including Acinetobacter baumannii (41\%), UTI was dominated by Enterobacteriaceae including Escherichia coli (19\%), SSI was dominated by A. baumannii (25\%), GI was dominated by Clostridium difficile (70\%), SST was dominated by E. coli (20\%) and Klebsiella pneumoniae (20\%), and OTH was dominated by K. pneumoniae (18\%) (Table 3).

Table 3. Microorganisms isolated in HAI in the ICU, 2007-2016.

\begin{tabular}{|c|c|c|c|c|c|c|c|c|}
\hline \multirow{2}{*}{$\begin{array}{l}\text { Number of HAI with } \\
\text { Microorganisms, All }\end{array}$} & \multirow{2}{*}{$N$} & \multirow{2}{*}{$\begin{array}{l}\text { BSI } \\
156\end{array}$} & \multirow{2}{*}{$\begin{array}{l}\text { PN } \\
174\end{array}$} & \multirow{2}{*}{$\begin{array}{c}\text { UTI } \\
54\end{array}$} & \multirow{2}{*}{$\begin{array}{c}\text { SSI } \\
32\end{array}$} & \multirow{2}{*}{$\begin{array}{l}\text { GI } \\
27\end{array}$} & \multirow{2}{*}{$\begin{array}{c}\text { SST } \\
25\end{array}$} & \multirow{2}{*}{$\begin{array}{c}\text { OTH } \\
18\end{array}$} \\
\hline & & & & & & & & \\
\hline & & $n(\%)$ & $n(\%)$ & $n(\%)$ & $n(\%)$ & $n(\%)$ & $n(\%)$ & $n(\%)$ \\
\hline Gram-positive cocci $(n)$ & 138 & 79 & 21 & 0 & 2 & 0 & 2 & 4 \\
\hline $\begin{array}{l}\text { Coagulase-negative } \\
\text { staphylococci }(\%)\end{array}$ & 70 & $69(44.2)$ & & & & & & $1(5.6)$ \\
\hline Staphylococcus aureus (\%) & 33 & $10(6.4)$ & $16(9.2)$ & & $2(6.3)$ & & $2(8.0)$ & $3(16.6)$ \\
\hline Enterococcus spp. (\%) & 32 & & $2(1.1)$ & & & & & \\
\hline Streptococcus pneumoniae (\%) & 3 & & $3(1.7)$ & & & & & \\
\hline Gram-positive other $(n)$ & 19 & 0 & 0 & 0 & 0 & 19 & 0 & 0 \\
\hline Clostridium difficile (\%) & 19 & & & & & $19(70.4)$ & & \\
\hline Enterobacteriaceae $(n)$ & 117 & 24 & 46 & 20 & 8 & 0 & 13 & 8 \\
\hline Escherichia coli $(\%)$ & 44 & $8(5.1)$ & $16(9.2)$ & $10(18.5)$ & $4(12.5)$ & & $5(20.0)$ & $1(5.6)$ \\
\hline Klebsiella pneumoniae (\%) & 35 & $8(5.1)$ & $14(8.0)$ & $2(3.7)$ & $1(3.1)$ & & $5(20.0)$ & $7(17.9)$ \\
\hline Enterobacter spp. (\%) & 18 & $4(2.6)$ & $7(4.0)$ & $4(7.4)$ & $1(3.1)$ & & $2(8.0)$ & \\
\hline Proteus spp. $(\%)$ & 11 & & $7(4.0)$ & $2(3.7)$ & $1(3.1)$ & & $1(4.0)$ & \\
\hline Seratia spp. (\%) & 5 & $3(1.9)$ & $1(0.6)$ & & $1(3.1)$ & & & \\
\hline Citrobacter spp. (\%) & 4 & $1(0.6)$ & $1(0.6)$ & $2(3.70)$ & & & & \\
\hline $\begin{array}{l}\text { Non-fermenting } \\
\text { gram-negative bacteria }(n)\end{array}$ & 165 & 33 & 96 & 10 & 13 & 0 & 6 & 7 \\
\hline Acinetobacter baumannii (\%) & 120 & $27(17.3)$ & $72(41.4)$ & $4(7.4)$ & $8(25.0)$ & & $4(16.0)$ & $5(27.7)$ \\
\hline Pseudomonas aeruginosa (\%) & 39 & $5(3.2)$ & $20(11.5)$ & $6(11.1)$ & $5(15.6)$ & & $1(4.0)$ & $2(11.1)$ \\
\hline Morganella morgannii (\%) & 3 & $1(0.6)$ & $1(0.6)$ & & & & $1(4.0)$ & \\
\hline Stenotrophomonas maltophilia (\%) & 2 & & $2(1.1)$ & & & & & \\
\hline Hemophilus spp. (\%) & 1 & & $1(0.6)$ & & & & & \\
\hline
\end{tabular}


Table 3. Cont.

\begin{tabular}{|c|c|c|c|c|c|c|c|c|}
\hline \multirow{2}{*}{$\begin{array}{l}\text { Number of HAI with } \\
\text { Microorganisms, All }\end{array}$} & \multirow{2}{*}{$N$} & BSI & PN & UTI & SSI & GI & SST & OTH \\
\hline & & 156 & 174 & 54 & 32 & 27 & 25 & 18 \\
\hline & & $n(\%)$ & $n(\%)$ & $n(\%)$ & $n(\%)$ & $n(\%)$ & $n(\%)$ & $n(\%)$ \\
\hline Other bacteria $(n)$ & 9 & 3 & 3 & 0 & 2 & 0 & 1 & 0 \\
\hline Other bacteria (\%) & 9 & $3(1.9)$ & $3(1.7)$ & & $2(6.3)$ & & $1(4.0)$ & \\
\hline Fungi $(n)$ & 31 & 6 & 5 & 11 & 3 & 5 & 0 & 0 \\
\hline Candida albicans (\%) & 28 & $6(3.8)$ & $5(2.9)$ & $11(20.4)$ & $1(3.1)$ & $5(18.5)$ & & \\
\hline Candida glabrata (\%) & 2 & & & & $2(6.3)$ & & & \\
\hline Candida krusei (\%) & 1 & & & & & & & \\
\hline Viruses $(n)$ & 2 & 0 & 0 & 0 & 0 & 2 & 0 & 0 \\
\hline Adenovirus & 1 & & & & & $1(3.7)$ & & \\
\hline Norovirus & 1 & & & & & $1(3.7)$ & & \\
\hline
\end{tabular}

N: number; pneumonia (PN); blood stream infections (BSI); urinary tract infection (UTI); Gastrointestinal (GE); Surgical site infection (SSI); skin and soft tissue infection (SST); Other infections (OTH).

The average time between admission of a patient to the ICU and emergence of HAI was 22.9 days (median 17 days) and was statistically significantly different for each type of HAI ( $p<0.001)$. The average duration of an ICU patient's stay was 28.2 days (median 17 days). Patients with HAIs stayed in the ICU statistically significantly longer than those without HAIs $(p<0.001)$ (Table 4 ).

Table 4. Days until HAI onset, ICU length of stay, mortality related to the microorganism that gave rise to HAI in the ICU in the years 2007-2016.

\begin{tabular}{|c|c|c|c|c|c|}
\hline \multirow{2}{*}{ Infection Types } & \multirow[t]{2}{*}{$N$} & \multicolumn{2}{|c|}{$\begin{array}{l}\text { Average Number of Days between } \\
\text { Admission to HAI Emergence }\end{array}$} & \multicolumn{2}{|c|}{$\begin{array}{c}\text { Average Number of Days of } \\
\text { Patient's Stay in ICU }\end{array}$} \\
\hline & & $\begin{array}{l}\text { Pooled Mean } \\
\quad(95 \% \text { CI })\end{array}$ & SD & $\begin{array}{l}\text { Pooled Mean } \\
\quad(95 \% \mathrm{CI})\end{array}$ & SD \\
\hline \multicolumn{6}{|c|}{ type HAI } \\
\hline Patients without HAI & 1337 & $\mathrm{n} / \mathrm{a}$ & $\mathrm{n} / \mathrm{a}$ & $19.0(17.9-20.1)$ & 21.1 \\
\hline Pneumonia & 183 & $19.2(16.7-21.7)$ & 17.3 & $51.5(45.8-57.2)$ & 39.0 \\
\hline Blood stream infections & 159 & $24.1(20.2-27.9)$ & 24.7 & $49.2(42.9-55.6)$ & 39.7 \\
\hline Urinary tract infection & 54 & $30.4(23.9-36.8)$ & 23.6 & $62.3(50.9-73.6)$ & 41.2 \\
\hline Gastrointestinal & 38 & $13.7(11.2-16.3)$ & 7.7 & $42.2(29.8-55.0)$ & 37.8 \\
\hline Surgical site infection & 33 & $26.3(21.4-31.2)$ & 13.8 & $67.0(50.7-83.3)$ & 45.9 \\
\hline Skin and soft tissue infection & 25 & $42.0(29.2-54.8)$ & 31.1 & $73.2(55.7-90.6)$ & 41.3 \\
\hline Other infections & 18 & $14.3(7.5-21.1)$ & 13.7 & $34.5(23.6-51.6)$ & 28.2 \\
\hline Total & 1847 & $22.9(21.0024 .8)$ & 21.0 & $28.2(26.8-29.7)$ & 31.5 \\
\hline Materiality ANOVA $(p)$ & $\mathrm{n} / \mathrm{a}$ & $p<0.001$ & $\mathrm{n} / \mathrm{a}$ & $p<0.001$ & $\mathrm{n} / \mathrm{a}$ \\
\hline \multicolumn{6}{|c|}{ selected aetiological HAI factors } \\
\hline Clostridium difficile & 19 & $12.4(8.4-16.4)$ & 8.3 & $35.5(19.2-51.8)$ & 32.7 \\
\hline Staphylococcus aureus & 33 & $19.0(16.1-22.0)$ & 16.2 & $50.3(33.4-80.6)$ & 40.8 \\
\hline Staphylococcus CNS & 70 & $20.8(16.4-32.5)$ & 28.5 & $47.5(35.7-58.5)$ & 39.5 \\
\hline Enterobacter spp. & 18 & $22.7(15.6-23.1)$ & 15.7 & $58.3(42.0-71.9)$ & 43.3 \\
\hline Acinetobacter baumannii & 120 & $23.6(17.6-29.6)$ & 19.7 & $40.0(37.2-51.0)$ & 22.7 \\
\hline Escherichia coli & 44 & $24.3(18.0-29.0)$ & 15.3 & 53.7 (46.3-61.1) & 40.6 \\
\hline Klebsiella pneumoniae & 35 & $27.1(13.2-37.5)$ & 25.3 & $67.8(46.4-77.8)$ & 45.1 \\
\hline Candida albicans & 28 & $31.5(23.0-39.9)$ & 26.2 & $57.5(45.9-69.1)$ & 38.3 \\
\hline Pseudomonas aeruginosa & 39 & $35.9(24.4-44.4)$ & 29.7 & $59.7(42.3-73.5)$ & 42.8 \\
\hline Enterococcus spp. & 32 & $36.2(25.5-45.6)$ & 30.2 & $66.3(52.3-80.2)$ & 43.1 \\
\hline
\end{tabular}

$\mathrm{N}$ : number; Me: median; SD: standard deviation; $95 \%$ confidence interval (CI) for mean; $\mathrm{n} / \mathrm{a}$ : not applicable; CNS: coagulase-negative staphylococci.

The number of days that elapsed before the infection and the duration of a patient's stay in the ICU were different for each species (or genus) of microorganism. In patients with HAIs, the mortality rate was $42.5 \%$, and in the non-infected group, the mortality rate was $45.3 \%$; this difference was not 
statistically significant $(p=0.296)$. The index calculated for each species (species) of the microorganism showed that the highest mortality rate was observed in patients with fungal infections (Table 5).

Table 5. Antimicrobial resistance in HAI in the ICU, 2007-2016.

\begin{tabular}{|c|c|c|c|c|}
\hline Microbial Genus/Species & $\begin{array}{l}\text { Number of } \\
\text { Isolates, Total }\end{array}$ & $\begin{array}{l}\text { Number of } \\
\text { Isolates with } \\
\text { Resistance } \\
\text { Mechanism }\end{array}$ & Resistant Codes & \\
\hline & & $n(\%)$ & & $n(\%)$ \\
\hline \multicolumn{5}{|c|}{ Gram-positive cocci } \\
\hline Staphylococcus aureus & 33 & $10(30.3)$ & $\begin{array}{l}\text { MRSA } \\
\text { MLSB }\end{array}$ & $\begin{array}{c}7(21.1) \\
3(9.1) \\
\end{array}$ \\
\hline $\begin{array}{l}\text { Coagulase-negative } \\
\text { staphylococci }(\%)\end{array}$ & 70 & $30(42.8)$ & $\begin{array}{l}\text { MRSA } \\
\text { MLSB }\end{array}$ & $\begin{array}{c}25(35.7) \\
5(7.1)\end{array}$ \\
\hline Enterococcus spp. & 32 & $10(31.2)$ & HLAR & $10(31.2)$ \\
\hline \multicolumn{5}{|c|}{ Enterobacteriaceae } \\
\hline Escherichia coli & 44 & $3(6.8)$ & ESBL & $3(6.8)$ \\
\hline Klebsiella pneumoniae & 35 & $10(28.6)$ & $\begin{array}{l}\text { MBL } \\
\text { ESBL }\end{array}$ & $\begin{array}{c}2(5.7) \\
8(22.9)\end{array}$ \\
\hline \multicolumn{5}{|c|}{ Non-fermenting gram-negative bacteria } \\
\hline $\begin{array}{l}\text { Acinetobacter baumannii. } \\
\text { Enterobacter spp. }\end{array}$ & $\begin{array}{c}120 \\
18\end{array}$ & $\begin{array}{c}5(4.1) \\
3(16.6)\end{array}$ & $\begin{array}{l}\text { MBL } \\
\text { ESBL }\end{array}$ & $\begin{array}{c}5(4.1) \\
3(16.6)\end{array}$ \\
\hline
\end{tabular}

MRSA: methicillin-resistant Staphylococcus aureus; ESBL: extended beta-lactamase produced; HLAR: high-level aminoglycoside resistance; MBL: metalo-beta lactamase.

Antibiotic resistance mechanisms were identified for the most common microorganisms (Table 5). Antibiotic susceptibility analysis was performed for the three most common Gram-negative organisms. Acinetobacter baumannii strains were characterized by high rates of resistance to many groups of antibiotics, $87 \%$ of which were extensive-drug resistant (XDR) strains. Similarly, $25 \%$ of K. pneumoniae strains were included in XDR, and $17 \%$ were multi-drug resistant (MDR). Pseudomonas aeruginosa strains were mostly antibiotic-sensitive, with resistance affecting $30 \%$ of the strains classified as MDR strains (Table 6).

Table 6. Resistance to a selected group of antibiotics and type of multi-drug resistance in the ICU in the years 2007-2016.

\begin{tabular}{|c|c|c|c|}
\hline Antibiotic Name & $\begin{array}{c}\text { Acinetobacter } \\
\text { baumannii }(N=120)\end{array}$ & $\begin{array}{c}\text { Pseudomonas } \\
\text { aeruginosa }(N=39)\end{array}$ & $\begin{array}{c}\text { Klebsiella } \\
\text { pneumoniae }(N=44)\end{array}$ \\
\hline \multicolumn{4}{|c|}{ Aminoglycosides (\%) } \\
\hline Gentamicin & 63.7 & 25.0 & 25.0 \\
\hline Tobramycin & 81.1 & 10.0 & 33.3 \\
\hline Amikacin & 95.6 & 15.0 & 25.0 \\
\hline Netilmicin & 88.4 & 20.0 & 16.6 \\
\hline \multicolumn{4}{|c|}{ Antipseudomonal carbapenems (\%) } \\
\hline Imipenem & 30.4 & 35.0 & 0.0 \\
\hline Mero penem & 33.3 & 20.0 & 8.3 \\
\hline \multicolumn{4}{|c|}{ Extended-spectrum cephalosporins (\%) } \\
\hline Ceftazidime & 98.1 & 15.0 & 41.6 \\
\hline Cefepime & 94.5 & 25.0 & 16.6 \\
\hline Cefotaxime & 99.3 & 75.0 & 58.3 \\
\hline Ceftriaxone & 97.8 & 65.7 & 66.6 \\
\hline
\end{tabular}


Table 6. Cont.

\begin{tabular}{|c|c|c|c|}
\hline Antibiotic Name & $\begin{array}{c}\text { Acinetobacter } \\
\text { baumannii }(N=120)\end{array}$ & $\begin{array}{c}\text { Pseudomonas } \\
\text { aeruginosa }(N=39)\end{array}$ & $\begin{array}{c}\text { Klebsiella } \\
\text { pneumoniae }(N=44)\end{array}$ \\
\hline \multicolumn{4}{|c|}{ Antipseudomonal fluoroquinolones (\%) } \\
\hline Ciprofloxacin & 97.8 & 10.0 & 41.6 \\
\hline Levofloxacin & 95.6 & 10.0 & 8.3 \\
\hline \multicolumn{4}{|c|}{ Penicillins (piperacillin) (\%) } \\
\hline Piperacillin & 0.0 & 20.0 & 0.0 \\
\hline \multicolumn{4}{|c|}{ Penicillins and b-lactamase inhibitors (\%) } \\
\hline Ticarcillin clavulanic acid & 0.0 & 20.0 & 16.6 \\
\hline Piperacillin tazobactam & 98.7 & 0.5 & 25.0 \\
\hline Ampicillin Sulbactam & 57.8 & 0.0 & 0.0 \\
\hline \multicolumn{4}{|c|}{ Cephalosporins and inhibitors (\%) } \\
\hline Cefoperazone sulbactam & 11.5 & 15.0 & 16.6 \\
\hline \multicolumn{4}{|c|}{ Polymyxins (\%) } \\
\hline Colistin & 1.2 & 0.5 & 0.0 \\
\hline Polymyxin B & 0.0 & 0.0 & 0.0 \\
\hline \multicolumn{4}{|c|}{ Type of resistance } \\
\hline Sensitive (\%) & 1.4 & 65.0 & 58.3 \\
\hline MDR (\%) & 11.5 & 30.0 & 16.6 \\
\hline XDR $(\%)$ & 86.9 & 0.0 & 25.0 \\
\hline PDR (\%) & 0.0 & 0.0 & 0.0 \\
\hline
\end{tabular}

The number of HAIs caused by the A. baumannii strains significantly fluctuated in the years under studyexamined herein, most frequently in 2008-2009, and a rapid trend of A. baumannii resistance to carbapenems was observed (Table 7).

Table 7. Compilation of the number of Acinetobacter baumannii isolates resistant to carbapenems in the years 2007-2016.

\begin{tabular}{cccc}
\hline \multirow{2}{*}{ Year } & Number of Acinetobacter baumannii Isolates & Imipenem & Meropenem \\
\cline { 3 - 4 } & & $\boldsymbol{n} \mathbf{( \% )}$ & $\boldsymbol{n} \mathbf{( \% )}$ \\
\hline 2007 & 11 & $1(9.1)$ & $0(0.0)$ \\
2008 & 26 & $2(7.7)$ & $1(3.8)$ \\
2009 & 26 & $3(11.5)$ & $2(7.7)$ \\
2010 & 7 & $1(14.4)$ & $4(57.1)$ \\
2011 & 3 & $1(33.3)$ & $1(33.3)$ \\
2012 & 13 & $6(46.2)$ & $9(69.2)$ \\
2013 & 4 & $1(25.0)$ & $3(75.0)$ \\
2014 & 11 & $7(63.6)$ & $7(63.6)$ \\
2015 & 13 & $10(76.9)$ & $10(76.9)$ \\
2016 & 6 & $4(66.7)$ & $3(50.0)$ \\
totality & 120 & $36(30.4)$ & $40(33.3)$ \\
\hline
\end{tabular}

$n(\%)$ : number and percentage of strains resistant to imipenem and meropenem.

\section{Discussion}

The results of our single-centre study are among the very few available in Poland. The study has a limited character because observational single-centre studies cannot be treated as a benchmark for all the population of Polish patients treated in ICUs. Therefore, the results of the study should be treated as an information basis on which preventive strategy of HAIs should be built accompanied by active methods of supervision aimed at improving the safety of patients treated in ICUs. It is not easy 
to assess the condition of a critically ill patient treated in ICU and it is not always possible because of the dynamics of pathological changes and the intensity of treatment. Thus, the next limitation for the studies is lack of proper classification of patients' condition based on APACHEII and SOFA scale. Lack of such an evaluation in the group of 1849 patients treated in ICUs within 10 years resulted in not including the results of several hundred patients into the total analysis. That is why the study is focused mainly on evaluation of various types of HAIs and their determinants. In our ICU, the incidence of HAIs was $28 \%$. The results obtained were similar to the results obtained in the meta-analysis performed by Allegranzi et al. [4], in which the incidence was 30\%. In one of the single-centre studies conducted in Poland by Wieder-Huszla [2] in 2010, the incidence of HAIs was 24\%. Additionally, Kübler et al. [3] conducted another single-centre study in Poland in 2012 and reported the incidence of HAIs as $24 \%$. In the point prevalence survey (PPS) based on morbidity and performed in Poland in 2011-2013, morbidity in the ICU was 35\%. In the literature, lower HAI incidence rates have also been reported, ranging from $9 \%$ to $16 \%$ [5,15]. In our ICU, the mortality of patients with healthcare-associated infections was $12 \%$. The result is similar to the mortality rate reported in the 2007 ECDC report, with an average mortality rate ranging to $15 \%$ [1]. Agodi et al. [16] detected $18 \%$ mortality in the ICU examined. The average age of patients treated in the ICU was 58 years, with a predominance of men, and the ratio of men to women was 1.55. In other European countries, the average age of patients was 61, and the sex male-to-female ratio was 1.56 [1]. In Rosenthal et al. [17], the average age of patients was 60 years. This analysis indicated that the patients in the analysed ward were younger, which may imply a worse health status in the Polish population and an earlier incidence of the diseases than in the compared countries. In our study, the duration of a patient's stay in the ICU was 19 days for patients without HAIs and as many as 55 days for patients with HAIs. In European countries, the length of ICU stays was between 8 and 13 days (the average amounting to 10 days) [1]. The extended stay of patients in the examined ward may be related to a different healthcare organization in Poland as well as to the infrastructure of the hospital wards, whose managements are afraid of accepting and continuing the treatment of a patient in a difficult state of health [18]. For patients with HAI, their longer stays may be related to the location of the infection as well as to the species of infecting micro-organism and its susceptibility to antibiotics $[19,20]$.

Pneumonia was most frequently diagnosed, the incidence being $10 \%$. In the ECDC report, which included data from 2007 [1], the average incidence was lower at 7\%, but the differences between European countries were large and ranged from 3\% to 36\%. In a subsequent ECDC report in 2014, the average incidence was 6\% [21]. In Rosnthal et al. [22] report from 50 countries the VAP incidence ranged between 0.9 and 13.1 per 1000 ventilator-days. Ventilator associated pneumonia density in the American CDC NHSN program from 2012 was on average 0.9 per 1000 ventilator-days [23]. In the present study, the IAP density was 15 in 1000 days with intubation. In European countries, in a study published in 2012, the mean density of IAP was 8 in 1000 with intubation [1]. In Rosenthal et al. [17] the IAP density in different ICUs ranged from 10 to 53 per 1000 days with a respirator. In another study of 43 countries, 17 patients were treated with a respirator [24]. In ECDC reports published in the last few years the mean device-adjusted rate was 10 IAP episodes per 1000 intubation days and varied between 3 (UK-Scotland) and 16 (Italy) in 2014; 2 (Luxembourg) and 18 (Italy) in 2015 [25,26]. In a single-centre study conducted in Poland in the ICU in 2012/2013/2014, the prevalence of a IAP was $11 / 9 / 10$ for 1000 days with intubation, respectively [27]. The average time between the admission to the onset of PN in our study was 19 days (median 15 days). In an ECDC report, the mean incubation time from ICU admission to the onset of pneumonia was 16 days (median 10 days) [1]. In our study, the duration of hospitalization for PN patients was 52 days on average (median 38 days), which means a significant, almost three-fold increase in hospitalizations in comparison with that of non-infected patients. Another study conducted in Poland confirmed the long 36-day hospitalization of PN patients [28]. In the ECDC study, the duration of hospitalization of patients with PN was approximately 3.5 times higher than that of patients without PN [1]. In the further part of our own study, PN subtypes were identified (based on diagnostics), with PN4 dominating (145 cases (79\%)). Similar 
evidence can be found in the ECDC report, in which there were approximately $50 \%$ of PN4. However, there were significant differences between the countries surveyed [1]. A. baumannii (41\%), P. aeruginosa $(12 \%)$, S. aureus and E. coli $(9 \%)$ and K. pneumoniae (8\%) were predominant in our study. For comparison, in the ECDC study pneumonia was most commonly caused by the following: P. aeruginosa (19\%), S. aureus (16\%), and E. coli (9\%) [1]. In another ECDC study conducted in 2011-2012 for infections in European countries, PN was predominant in Pseudomonas aeruginosa $(17 \%)$, Staphylococcus aureus (13\%), and Klebsiella spp. (11\%) [29]. The ECDC study found the same microorganisms in a similar proportion, the only difference being a more frequent occurrence of Acinetobacter baumannii in our ICU. Acinetobacter baumannii also predominated in PNs detected in ICU in other Polish studies [2,27]. In a study by Chmielarczyk et al. [30], Acinetobacter baumannii induced $79 \%$ of PN. It can therefore be concluded that similar PN aetiological factors are present in regions of Poland and other European countries. The mortality rate in our study was $45 \%$.

The second-most common type of HAI was bloodstream infections (BSIs), with a 9\% incidence. In the 2007 ECDC report, the incidence in European countries ranged from $2 \%$ to $5 \%$ (on average $4 \%$ ) [1]. In a subsequent ECDC report published in 2015, the incidence was 3\% [21]. In our study, the density of catheter-associated bloodstream infections (CLA-BSI) was 8 per 1000 central-line days. As the 2012 European ECDC report reported, the density of bloodstream infections with CLA-BSI varied between 2 and 5 in 1000 central-line days in Europe (the average being 3 in 1000 CVC days) [1]. Comparing our findings with those from European programmes, it could be observed that bloodstream infections are twice as common in our study in the incidence rates and densities. In another study conducted in 55 ICUs in developing countries, the density of bloodstream infections with CLA-BSI ranged from 8 to 19 per 1000 central-line days (average of 13 per 1000 central-line days) [17]. Rosnthal et al. [22] in the report INICC 2010-2015 from 50 countries CLA-BSI ranges between 0.8 and 4.1 per 1000 central-line days. CLA-BSI density obtained in the American CDC NHSN program in 2012 was on average 1.8 per 1000 central-line days [23]. In ECDC report from 2014 the mean device-adjusted rate was: 2.4 CLA-BSI episodes per 1000 central-line days [26]. In ECDC from 2015 the mean device-adjusted rate was 3.6 CLA-BSI episodes per 1000 central-line days and varied between 1 (Luxembourg) and 8 (Slovakia) [25]. The obtained results classified the occurrence of catheter-associated bloodstream infections in Poland at the lower border of developing countries with respect to CLA-BSI prevalence. In another study of 43 countries, the density was 5 per 1000 CVC days [24]. The average time between admission to BSI was 24 days (median 16 days). In the ECDC report [1], the onset of BSIs occurred on day 20 (median 14 days). In our BSI study, the following physiological flora were dominant in BSIs: Coagulase-negative staphylococci $(44 \%)$, A. baumannii $(17 \%)$, and S. aureus $(6 \%)$. The results obtained were comparable to those reported in the 2012 ECDC study for CLA-BSIs [1], where coagulase-negative staphylococci was present in $41 \%$, S. aureus was present in $13 \%$, and Enterococcus spp. was present in $12 \%$. In the 2017 ECDC report [26], coagulase-negative staphylococci were similarly dominant. In a study by Chmielarczyk et al. [30] conducted in Poland in 2016, Acinetobacter baumannii was detected in $17 \%$ cases in BSI in ICU. The mortality rate for BSIs was $45 \%$. In a study carried out in Poland by Wójkowska-Mach et al. [31], the mortality rate related to primary blood infection in ICU was higher and amounted to 19\%. In the 2007 ECDC report, mortality in BSIs averaged 33\% (21\% to 40\%) [1].

The third-most common form of hospital infection was urinary tract infections. In our study, the incidence rate was $3 \%$, and the density of UTI with catheter-associated urinary tract infection (CA-UTI) amounted to 3 per 1000 catheter days. In a single-centre study conducted in Poland by Duszyńska et al. [32], the incidence rate was 7\%. In the 2007 ECDC report, the incidence in European countries was $7 \%$, and the density was 1 to 21 (with an average of 5 per 1000 catheter days) [1]. In Rosnthal et al. [22] INICC 2010-2015 raport from 50 countries CA-UTI ranges from 1.7 to 5.1 per 1000 catheter days. CA-UTI Density in American CDC NHSN program from 2012 was on average 1.7 per 1000 catheter days [23]. In a study conducted in 2006 in 55 intensive care units in developing countries, the incidence of CA-UTI ranged from 2 to 13 per 1000 catheter days [17]. In a study of 43 countries conducted in 2014, the density was 5 per 1000 catheter days [24]. The lower CA-UTI 
scores, which we obtained, may be due to the weakness of the HAI surveillance structures and little experience in this field [18]. In ECDC from 2014 and 2015 the mean device-adjusted rate was 4 CA-UTI episodes per 1000 catheter days [25,26]. In the ECDC report [1], the onset of UTI was on the twenty-first day of residence (median: 15 days). In the ICU under our examination, the onset of UTI was on average 30 days (median: 24 days), and the average hospitalization time was 62 days (median 54 days), which implies a significant increase in hospitalization for non-infected patients. In our study, UTIs was dominated by the following microbiota: Candida albicans (20\%), E. coli (19\%), and P. aeruginosa (11\%). The microbiota detected in our study was similar to the one from 2007 ECDC report: Escherichia coli (25\%), C. albicans (17\%), and Enterococcus spp. (18\%). The UTI mortality rate was $41 \%$. In the European study, a large variation—-between $7 \%$ and $37 \%$-in UTI mortality rates was observed [1].

In the ICU in our study, other types of healthcare-associated infections were present: GI (2\%), SSI $(2 \%)$, SST $(1 \%)$, and OTH (1\%). In a multicentre prevalence-based study, healthcare-associated infections were detected in the ICU in slightly different percentages: GI (morbidity $4 \%$ ), SSI (9\%), SST $(3 \%)$ and OTH (4\%) [10].

In the present study, the prevalence's of multidrug resistance (MDR) strains was maintained at a similar level in particular years: Staphylococcus aureus MRSA (21\%), MRSE coagulase-negative staphylococci (36\%), ESBL Escherichia coli (7\%), Klebsiella spp. (23\%), and Enterococcus spp. (31\%). In the 2007 ECDC report, the findings were as follows: Staphylococcus aureus MRSA (35\%), coagulase-negative staphylococci (83\%), E. coli ESBL (12\%), Klebsiella spp. (23\%) [1,21], Escherichia coli ESBL (12\%), Klebsiella spp. (26\%), Staphylococcus aureus MRSA (25\%), and Enterococcus spp. (25-50\%) [21]. However, in other studies, a clear trend was observed for antimicrobial resistance, especially among Gram-negative bacilli [19,20,29]. The 2017 ECDC report [26], provided the following results: 25\% Staphylococcus aureus MRSA, 17\% ESBL E. coli, 44\% Klebsiella spp., and 44\% Enterobacter spp.

Clinical specimens from HAI patients were isolated from multidrug resistance (MDR) strains, which implies insufficient sensitivity to at least one antibiotic from three or more antimicrobial drug classes active against the species. In our study, 30\% of Staphylococcus aureus strains showed methicillin resistant (MRSA) strains, but no vancomycin resistant strains (VRSA) were detected. The stabilization of MRSA infections and the small percentage of Gram $(+)$ microorganisms resistant to vancomycin-resistant bacteria has been observed in the literature [19]. A literature analysis shows that the growth in resistance to antibiotics of Gram (-), especially K. pneumoniae $[19,20,22,26,29]$ is on the increase. In the present study, the percentage of K. pneumoniae resistant to third-generation cephalosporins ranged from $17 \%$ to $67 \%$. In a study conducted under the PPS (ECDC) programme in Europe in 2012, K. pneumoniae, isolated from invasive infections, showed, on average, a $26 \%$ resistance to third-generation cephalosporins (from 2\% to 79\%); in Poland, K. pneumoniae showed a $73 \%$ resistance [29].

While analysing the antimicrobial resistance of antibiotics, Acinetobacter baumannii was found to be $87 \%$ extensive-drug resistant (XDR), which means there was insensitivity to one or more antibiotics in all but two or fewer classes of antibiotics. Based on a review of the literature and the observed trends of resistance among Acinetobacter baumannii, the results obtained in our own study were comparable to the results of reports from European countries, where the microorganism displayed a wide spectrum of resistance [29]. In a study by Chmielarczyk et al. [30], extensive drug resistant strains (XDR) accounted for $80.8 \%$ of the tested isolates and were detected in HAI patients in the ICU. Therefore, monitoring drug resistance of $A$. baumannii strains in ICUs is vital with respect to planning specific infection control measures to prevent the spread of MDR and XDR to other patients.

According to Różańska et al. [33], in Poland, infection control programmes are a challenge for the future, and their implementation requires increasing the awareness of both medical staff and hospital management. The presented results constitute some of the research based on the method of active, continuous surveillance of infections in the ICU in Poland. The results obtained confirm the reliability of surveillance conducted in the ICU under examination. The results can provide a basis for 
comparing the epidemiological situation in other ICUs, which may accordingly contribute to effective HAI prevention.

\section{Conclusions}

(1) In the ICU study, pneumonia and bloodstream infections were the most common.

(2) Acinetobacter baumannii strains were most commonly isolated from clinical specimens collected from HAI patients.

(3) The number of Acinetobacter baumannii infections did not have a tendency during the period considered.

(4) The strains of Acinetobacter baumannii mostly showed resistance to many antibiotic groups.

(5) There was a trend of an increasing resistance of Acinetobacter baumannii to carbapenems.

(6) Action is needed to limit the spread of these strains.

Acknowledgments: Ethical approval: this is a completed retrospective study and the paper does not report on primary research. The data collected were not set up as a study or research project, but as part of an infection control programme. The paper does not report on the use of experimental or new protocols. Funding Source: This research did not receive any specific grant from funding agencies in the public, commercial, or not-for-profit sectors. Financial Disclosure: The authors have no financial relationships relevant to this article to disclose.

Author Contributions: Małgorzata Kołpa conceptualized and designed the study, analysed and interpreted the data, drafted the manuscript; Marta Wałaszek collected data, carried out the initial analyses and statistical analysis and analyzing the literature; Agnieszka Gniadek interpreted data, drafted the manuscript and gave final approval of the version to be published, the corresponding author; Zdzisław Wolak collected data, carried out the initial analyses; Wiesław Dobroś interpreted data and drafted the manuscript.

Conflicts of Interest: The authors declare no conflict of interest.

\section{Abbreviations}

$\begin{array}{ll}\text { HAIs } & \text { Healthcare-Associated Infections } \\ \text { DA-HAI } & \text { Device-Associated Healthcare-Associated Infection } \\ \text { ICU } & \text { Intensive Care Unit } \\ \text { ECDC } & \text { European Centre for Disease Prevention and Control } \\ \text { PN } & \text { Pneumonia } \\ \text { IAP } & \text { Intubation Associated Pneumonia } \\ \text { VAP } & \text { Ventilator Associated Pneumonia } \\ \text { PN2 } & \text { Non-protected sample with quantitative culture } \\ \text { PN3 } & \text { Alternative microbiological criteria } \\ \text { PN4 } & \text { Sputum bacteriology or non-quantitative } \\ \text { PN5 } & \text { No microbiological documentation } \\ \text { BSI } & \text { Bloodstream Infection } \\ \text { CVC } & \text { Central Venous Catheter } \\ \text { CLA-BSI } & \text { Central Line-Associated Bloodstream Infection } \\ \text { BSI-C } & \text { Vascular Catheter-Related } \\ \text { CRI1-CVC } & \text { Primary BSI local Catheter Related Infection } \\ \text { CRI2-CVC } & \text { Primary BSI general Catheter Related Infection } \\ \text { CRI3-CVC } & \text { Primary BSI microbiologically confirmed Catheter Related } \\ \text { BSI UO } & \text { Primary BSI of Unknown Origin } \\ \text { BSI-S } & \text { Secondary BSI to another infection: DIG, PUL, SSI } \\ \text { UTI } & \text { Urinary Tract Infections } \\ \text { CA-UTI } & \text { Catheter-Associated UTI } \\ \text { UTI-A } & \text { Microbiologically confirmed symptomatic } \\ \text { UTI-B } & \text { Not microbiologically confirmed symptomatic urinary tract infection } \\ \text { GI } & \text { Gastrointestinal } \\ \text { GI-CDI } & \text { Clostridium difficile infection } \\ \text { GI-GE } & \text { Gastroenteritis } \\ \text { SSI } & \text { Surgical Site Infection } \\ & \end{array}$




\begin{tabular}{ll} 
SSI-D & Deep incisional \\
SSI-O & Organ/space \\
SSI-S & Superficial incisional \\
SST & Skin and Soft Tissue Infection \\
SST-DECU & Decubitus ulcer \\
SST-SKIN & Skin infection \\
SST-ST & Soft tissue \\
CNS & Central Nervous System Infections \\
EENT & Eye, Ear, Nose, Throat or Mouth Infection \\
LRI & Lower Respiratory Tract \\
SYS & Systemic Infections \\
OTH & Other Infections \\
BAL & Broncheoalveolar Lavage \\
CFU & Colony Forming Unit \\
XDR & Extensive Drug Resistant \\
PDR & Pan Drug Resistant \\
MDR & Multi Drag Resistant \\
EUCAST & European Committee on Antimicrobial Susceptibility Testing \\
$p$ & Significance level \\
Me & Median \\
SD & Standard Deviation \\
95\% CI & Confidence Interval \\
OR & Odds ratio \\
DUR & Device Use Ratio \\
INICC & International Nosocomial Infection Control Consortium \\
NHSN & National Halthcare Safety Network in USA \\
APACHEII & Acute Physiology And Chronic Health Evaluation II score \\
SOFA & Sepsis-Related Organ Failure Assessment score \\
\hline &
\end{tabular}

\section{References}

1. European Center for Disease Prevention and Control. Surveillance of Healthcare-Associated Infections in Europe, 2007; ECDC: Stockholm, Sweden, 2012; pp. 43-71. Available online: http://ecdc.europa.eu/en/ publications / Publications/120215SURHAI2007.pdf (accessed on 30 June 2017).

2. Wieder-Huszla, S. Monitorowanie zakażeń szpitalnych w oddziale intensywnej terapii medycznej. Ann. Acad. Med. Stetin. 2010, 56, 20-29. (In Polish) [PubMed]

3. Kübler, A.; Duszyńska, D.; Rosenthal, V.D.; Fleischer, M.; Kaiser, T.; Szewczyk, E.; Barteczko-Grajek, B. Device-associated infection rates and extra length of stay in an intensive care unit of a university hospital in Wroclaw, Poland: International Nosocomial Infection Control Consortium's (INICC) findings. J. Crit. Care 2012, 27, 105.e5-105.e10. [CrossRef] [PubMed]

4. Allegranzi, B.; Nejdar, S.B.; Castillejos, G.G.; Kilpatrick, C.; Kelley, E.; Mathai, E. Clean Care is Safer Care Team. In Report on the Burden of Endemic Health Care-Associated Infection Worldwide; A Systematic Review of the Literature; World Health Organization: Geneva, Switzerland, 2011, ISBN 9789241501507.

5. Simonetti, A.; Ottaiano, E.; Diana, M.V.; Onza, C.; Triassi, M. Epidemiology of hospital-acquired infections in an adult intensive care unit: Results of a prospective cohort study. Ann. Ig. 2013, 25, 281-289. (In Italian) [CrossRef] [PubMed]

6. European Center for Disease Prevention and Control. Point Prevalence Survey of Healthcare-Associated Infections and Antimicrobial Use in European Acute Care Hospitals_Protocol Version 4.3; ECDC: Stockholm, Sweden, 2012. Available online: http:/ / ecdc.europa.eu/en/publications/publications/0512-ted-pps-haiantimicrobial-use-protocol.pdf (accessed on 4 October 2017).

7. European Centre for Disease Prevention and Control. European Surveillance of Healthcare Associated Infections in Intensive Care Units-HAI-Net ICU Protocol, Version 1.02; ECDC: Stockholm, Sweden, 2015. Available online: https://ecdc.europa.eu/sites/portal/files/media/en/publications/ Publications/healthcare-associated-infections-HAI-ICU-protocol.pdf (accessed on 4 October 2016). 
8. Chacko, B.; Thomas, K.; David, T.; Paul, H.; Jeyaseelan, L.; Peter, J.V. Attributable cost of a nosocomial infection in the intensive care unit: A prospective cohort study. World J. Crit. Care Med. 2017, 6, 79-84. [CrossRef] [PubMed]

9. Bouadma, L.; Deslandes, E.; Lolom, I.; Le Corre, B.; Mourvillier, B.; Regnier, B.; Porcher, R.; Wolff, M.; Lucet, J.C. Long-term impact of a multifaceted prevention program on ventilator-associated pneumonia in a medical intensive care unit. Clin. Infect. Dis. 2010, 51, 1115-1122. [CrossRef] [PubMed]

10. Gords, B.; Vrijens, F.; Hulstaest, F.; Devriese, S.; Van de Sande, S. The 2007 Belgian national prevalence survey for hospital-acquired infections. J. Hosp. Infect. 2010, 75, 163-167. [CrossRef] [PubMed]

11. Iordanou, S.; Middleton, N.; Papathanassoglou, E.; Raftopoulos, V. Surveillance of device associated infections and mortality in a major intensive care unit in the Republic of Cyprus. BMC Infect. Dis. 2017, 17, 607. [CrossRef] [PubMed]

12. Prin, M.; Li, G. Complications and in-hospital mortality in trauma patients treated in intensive care units in the United States, 2013. Inj. Epidemiol. 2016, 4, 18. [CrossRef] [PubMed]

13. CDC Guidelines for Isolation Precautions: Preventing Transmission of Infectious Agents in Healthcare Settings. Available online: http://www.cdc.gov/ncidod/dhqp/pdf/isolation2007.pdf (accessed on 10 October 2017).

14. Sehulster, L.; Raymond, Y.W. Guidelines for Environmental Infection Control in Health-Care Facilities. Recommendations of CDC and the Healthcare Infection Control Practices Advisory Committee (HICPAC). Recomm. Rep. 2003, 52, 1-42.

15. Ling, M.L.; Apisarnthanarak, A.; Madriaga, G. The burden of healthcare-associated infections in Southeast Asia: A systematic literature review and meta-analysis. Clin. Infect. Dis. 2015, 60, 1690-1699. [CrossRef] [PubMed]

16. Agodi, A.; Auxilia, F.; Barchita, M.; Brusaferro, S.; D'Alessandro, A.; Grillo, O.C.; Montagna, M.T.; Pasaquarella, C.; Rrighi, E.; Tardivo, S.; et al. Trends, risk factor and outcomes of healthcare-associated infections within the Italian network SPIN-UTI. J. Hosp. Infect. 2013, 84, 52-58. [CrossRef] [PubMed]

17. Rosenthal, V.D.; Maki, D.G.; Salomato, R.; Moreno, C.A.; Mehta, Y.; Higuera, F.; Cuellar, L.E.; Arikan, O.A.; Abouqal, R.; Leblebicioglu, H.; et al. Device-associated nosocomial infections in 55 intensive care units of 8 developing countries. Ann. Intern. Med. 2006, 145, 582-591. [CrossRef] [PubMed]

18. Ider, B.E.; Adams, J.; Morton, A.; Whithy, M.; Clements, A. Infection control systems in transition: The challenges for post-Soviet Bloc countries. J. Hosp. Infect. 2012, 80, 277-287. [CrossRef] [PubMed]

19. Van Duijn, P.J.; Dautzenberg, M.J.; Oostdijk, E.A. Recent trends in antibiotic resistance in European ICUs. Curr. Opin. Crit. Care 2011, 17, 658-665. [CrossRef] [PubMed]

20. Prabaker, K.; Weinstein, R.A. Trends in antimicrobial resistance in intensive care units in the United States. Curr. Opin. Crit. Care 2011, 17, 472-479. [CrossRef] [PubMed]

21. European Center for Disease Prevention and Control. Antimicrobial Resistance and Healthcare-Associated Infection. In Annual Epidemiological Report 2014; ECDC: Stockholm, Sweden, 2015. Available online: http:/ / ecdc.europa.eu/en/publications / publications / antimicrobial-resistance-annual-epidemiologicalreport.pdf (accessed on 29 October 2017).

22. Rosenthal, V.D.; Al-Abdely, H.M.; El-Kholy, A.A.; Khawaja, S.A.A.; Leblebicioglu, H.; Mehta, Y.; Rai, V.; Hung, N.V.; Kanj, S.S.; Salama, M.F.; et al. International Nosocomial Infection Control Consortium report, data summary of 50 countries for 2010-2015: Device-associated module. Am. J. Infect. Control 2016, 44, 1495-1504. [CrossRef] [PubMed]

23. Dudeck, M.A.; Weiner, L.M.; Allen-Bridson, K.; Malpiedi, P.J.; Peterson, K.D.; Pollock, D.; Sievert, D.M.; Edwards, J.R. National Healthcare Safety Network (NHSN) report, data summary for 2012, Device-associated module. Am. J. Infect. Control 2013, 41, 1148-1166. [CrossRef] [PubMed]

24. Rosenthal, V.D.; Maki, D.G.; Mehta, Y.; Leblebicioglu, H.; Memish, Z.A.; Al-Mousa, H.H.; Balkhy, H.; Hu, B.; Alvarez-Moreno, C.; Medeiros, E.A.; et al. International Nosocomial Infection Control Consortium. International Nosocomial Infection Control Consortium (INICC) report, data summary of 43 countries for 2007-2012. Device-associated module. Am. J. Infect. Control 2014, 42, 942-956. [CrossRef] [PubMed]

25. European Center for Disease Prevention and Control. Healthcare-associated infection acquired in intensive care units. In Annual Epidemiological Report for 2015; ECDC: Stockholm, Sweden, 2017. 
26. European Center for Disease Prevention and Control. Healthcare-Associated Infections Acquired in Intensive Care Unit. In Annual Epidemiological Report 2016; ECDC: Stockholm, Sweden, 2016; Available online: https: / ecdc.europa.eu/en/publications-data/infections-acquired-intensive-care-unitsannual-report-2016 (accessed on 12 October 2017).

27. Duszyńska, W.; Rosenthal, V.D.; Dragan, B.; Wegrzyn, P.; Mazur, A.; Wojtyra, P.; Tomala, A.; Kübler, A. Ventilator-associated pneumonia monitoring according to the INICC project at one center. Anaesthesiol. Intensiv. Ther. 2015, 47, 34-39. [CrossRef] [PubMed]

28. Różańska, A.; Wójkowska-Mach, J.; Bulanda, M.; Heczko, P.B. Infection control in Polish medical wards-Data from the PROHIBIT project. Prz. Epidemiol. 2015, 69, 495-501.

29. European Centre for Disease Prevention and Control. Point Prevalence Survey of Healthcare Associated Infections and Antimicrobial Use in European Acute Care Hospitals; ECDC: Stockholm, Sweden, 2013; pp. 186-187. Available online: http:/ / ecdc.europa.eu/en/publications/publications/healthcare-associated-infectionsantimicrobial-use-pps.pdf (accessed on 30 October 2017).

30. Chmielarczyk, A.; Pilarczyk-Żurek, M.; Kamińska, W.; Pobiega, M.; Romaniszyn, D.; Ziółkowski, G.; Wójkowska-Mach, J.; Bulanda, M. Molecular epidemiology and drug resistance of Acinetobacter baumannii isolated from hospitals in southern Poland: ICU as risk factor for XDR strains. Microb. Drug Resist. 2016, 22, 328-335. [CrossRef] [PubMed]

31. Wójkowska-Mach, J.; Różańska, A.; Kuthan, R.; Bulanda, M.; Heczko, P. Śmiertelność związana z zakażeniami szpitalnymi. Analiza oparta na danych uzyskanych w ramach ogólnopolskiego programu kontroli zakażeń w 1998 roku. Prz. Epidemiol. 2000, 54, 281-290. (In Polish)

32. Duszyńska, W.; Rosenthal, V.D.; Dragan, B.; Szczęsny, A.; Woźnica, E.; Ulfik, K.; Ostrowska, E.; Litwin, A.; Kübler, A. Urinary tract infections in intensive care unit patients-A single-center, 3-year observational study according to the INICC project. Anaesthesiol. Intensiv. Ther. 2016, 48, 1-6. [CrossRef] [PubMed]

33. Różańska, A.; Wójkowska-Mach, J.; Bulanda, M. Surveillance of hospital acquired infections according to ECDC definitions in Polish hospital-A pilot study. Antimicrob. Resist. Infect. Control 2015, 4, P276. [CrossRef] 